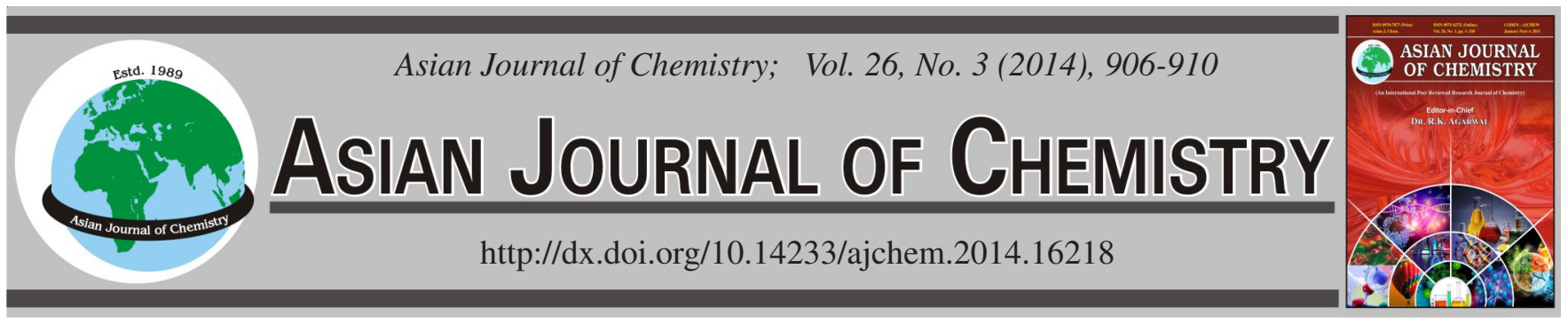

\title{
Compound-Specific Carbon Isotope Analysis of Polycyclic Aromatic Hydrocarbons in Water Using Solid-Phase Extraction Coupled with GC/C-IRMS
}

\author{
Lin Zhang*, YongtaO Zhang, Fuliang Liu and Jun LiU
}

Institute of Hydrogeology and Environmental Geology (IHEG), CAGS, Zhengding 050803, Hebei Province, P.R. China

*Corresponding author: Fax: +86 311 88017401; Tel: +86 311 88012335; E-mail: zhl5369@163.com

Received: 23 August 2013;

Accepted: 7 November 2013;

Published online: 30 January 2014;

AJC-14665

\begin{abstract}
A method using solid-phase extraction coupled with gas chromatography/combustion/isotope ratio mass spectrometry (GC/C-IRMS) for the compound-specific stable carbon isotope analysis of polycyclic aromatic hydrocarbons in aqueous phase was developed in this study. Parameters for the efficient extraction of polycyclic aromatic hydrocarbons from water samples were evaluated by SPE-GC/MS. The results indicated that individual polycyclic aromatic hydrocarbons in water can be effectively separated from unresolved complex mixtures by the proposed procedure. The mean recovery of the purification method for all compounds is $>80 \%$. The carbon isotope composition of polycyclic aromatic hydrocarbons can be measured with a standard deviation of $<1 \%$, for standard material extracts after purification. No significant isotopic fractionation was observed during the solid-phase extraction procedure. These findings indicate that the solidphase extraction-GC/C-IRMS method is well suited for the compound-specific carbon isotope analysis of polycyclic aromatic hydrocarbons in aqueous phase and can be used as a potential tool for the source identification of polycyclic aromatic hydrocarbons.
\end{abstract}

Keywords: Polycyclic aromatic hydrocarbon, Gas chromatography, Isotope ratio mass spectrometry.

\section{INTRODUCTION}

Interest in polycyclic aromatic hydrocarbons (PAHs) is increasing worldwide because of their known carcinogenic and mutagenic properties. Sixteen polycyclic aromatic hydrocarbons considered as priority by the American Environmental Protection Agency are naphthalene, acenaphthylene, acenaphthene, fluorene, anthracene, phenanthrene, fluoranthene, chrysene, benzo[a]anthracene, pyrene, benzo[k]fluoranthene, dibenzo[b,c]fluoranthene, benzo[a]pyrene, indeno[1,2,3cd] pyrene, dibenz $[\mathrm{a}, \mathrm{h}]$ anthracene and benzo $[$ ghi $]$ perylene ${ }^{1}$. Identification of polycyclic aromatic hydrocarbon sources though various techniques have been performed, including molecular marker approaches and mathematical modeling ${ }^{2-5}$. However, the development of new methods for polycyclic aromatic hydrocarbon source identification is still an ongoing concern. Compound-specific isotope analysis (CSIA) procedures can be exploited to trace polycyclic aromatic hydrocarbon contaminants in the environment to a particular source or responsible party ${ }^{6-12}$. O'Malley et al. ${ }^{7}$ performed the first measurement of carbon isotopes of individual polycyclic aromatic hydrocarbons from modern depositional environments and various anthropogenic sources. The CSIA of groundwater pollutants has become an important tool for identifying different sources of the same pollutant and to track natural attenuating processes in the subsurface. Changes in a contaminant's isotope signature with time, location, or both can reportedly be used as evidence for in situ degradation reactions ${ }^{13,14}$. Carbon isotope analysis of volatile organic compounds (VOCs) by CSIA has been reported in recent years ${ }^{15-20}$ and many researchers have applied this method in different environmental samples, such as aerosol, sediment and coal combustion particles ${ }^{21-24}$. To date, studies on analytical methods of determining the $\delta^{13} \mathrm{C}$ signatures of polycyclic aromatic hydrocarbon contaminants in water are limited. Given that the sensitivity of gas chromatographyisotope ratio mass spectrometry (GC-IRMS) is limited by the low ionization efficiency of the ion source and low abundance of stable isotopes, effective enrichment techniques of water must be used. To ensure high accuracy and precision of stable carbon isotope data of individual polycyclic aromatic hydrocarbons, separation and purification of polycyclic aromatic hydrocarbon fractions are often necessary before GC-C-IRMS measurement. Solid-phase extraction is a widely used enrichment method for semi-VOCs (SVOCs) from aqueous phase and solid samples because this method provides very low detection limits and good accuracy. Given that this method includes many phase-transfer steps, the influence on isotope composition must be evaluated. Accordingly, this study aimed to develop a method of measuring $\delta^{13} \mathrm{C}$ of polycyclic aromatic hydrocarbon contaminants in water and to evaluate the reproducibility and accuracy of the method. 


\section{EXPERIMENTAL}

All chemicals and reagents used were analytical grade and of the highest purity possible. Sixteen polycyclic aromatic hydrocarbons standards dissolved in $n$-hexane were purchased from Supelco (Bellefonte, PA, USA) and these standards are defined and nominated as priority pollutants by EPA, including naphthalene, acenaphthylene, acenaphthene, fluorene, phenanthrene, anthracene, fluoranthene, pyrene, benzo[a]anthracene, chrysene, dibenzo[b,c]fluoranthene, benzo[k]fluoranthen, benzo[a]pyrene, indeno[1,2,3cd]pyrene, dibenz $[a, h]$ anthracene and benzo[ghi]perylene.

All solvents including methanol, dichloromethane and $n$-hexane were HPLC grade and purchased from Dima Technology Inc. ( Richmond, USA). Ultrapure water (Milli, USA) was used to purify water, $\mathrm{He}(99.999 \%)$ and sodium sulfate (after drying at $450{ }^{\circ} \mathrm{C}$ for $4 \mathrm{~h}$ ). $\mathrm{C} 18$ solid-phase extraction columns $\left(250 \mathrm{mg} \times 2 \mathrm{~mL}^{-1}\right)$ were purchased from Supelco (St. Louis, MO, USA).

The GC-C-IRMS system consisted of a Trace DSQ (Thermo Finnigan, Germany) gas chromatograph equipped with a split/ splitless injector coupled with a MAT253 isotope ratio mass spectrometer (Thermo Finnigan, Bremen, Germany) through a GC-Combustion III interface (Thermo Finnigan). Helium was used as a carrier gas. Gas chromatography with a DB-5 fused silica capillary column $(30 \times 0.25 \mathrm{~mm}, 0.25 \mu \mathrm{m}$ film thickness) was used for separation. The combustion furnace temperature in the $\mathrm{GC}-\mathrm{C}$ interface was $960{ }^{\circ} \mathrm{C}$.

GC-MS system consisted of a gas chromatograph of Thermo Finnigan Trace GC (Thermo Electron Co., Waltham, MA, USA), equipped with an AI/AS3000 autosampler. A DB-5MS fusedsilica column was used with a DB-5 fused silica capillary column ( $30 \mathrm{~mm} \times 0.25 \mathrm{~mm}, 0.25 \mu \mathrm{m}$ film thickness; J \&W Scientific, USA).

Solid-phase extraction of water samples: Solid-phase extraction (SPE) is an effective technique of analyzing compounds adsorbed onto a solid support and subsequently removed either by thermal means or by using a solvent. The technique reduces the consumption of high-purity solvents, thereby reducing the cost and the need for solvent disposal. For solidphase extraction tests, standard solutions were prepared by dissolving a standard stock solution of polycyclic aromatic hydrocarbons at the same concentration of $20 \mathrm{mg} / \mathrm{L}$ in ultrapure water (Milli-Q) with a final volume of $1 \mathrm{~L}$. solid-phase extraction was performed using a standard solid-phase extraction system from Supelco (Bellefonte, PA, USA), connected with a vacuum pump. To avoid adsorption of polycyclic aromatic hydrocarbons in glassware, $5 \mathrm{~mL}$ of methanol was added to $1 \mathrm{~L}$ of water and the solution was mixed. $\mathrm{C} 18$ disks were first activated by wetting with $5 \mathrm{~mL}$ of $\mathrm{CH}_{2} \mathrm{Cl}_{2}$ followed by $5 \mathrm{~mL}$ of methanol and $5 \mathrm{~mL}$ of distilled water from the millipore system. Then, $1 \mathrm{~L}$ of water sample was applied at a speed of $10 \mathrm{~mL} \mathrm{~min}{ }^{-1}$ without letting the disk become dry. The disks were then dried under a vacuum for $10 \mathrm{~min}$. Elution was performed with $2 \mathrm{~mL}$ of $n$-hexane and $5 \mathrm{~mL}$ of $\mathrm{CH}_{2} \mathrm{Cl}_{2}$ at a flow rate of $2-3 \mathrm{~mL} \mathrm{~min}^{-1}$ thrice. The extract was dried over anhydrous $\mathrm{Na}_{2} \mathrm{SO}_{4}$ and concentrated to approximately $0.7 \mathrm{~mL}$ until dry under a gentle stream of nitrogen in a water bath at $40{ }^{\circ} \mathrm{C}$. The resulting product was then transferred to a $1 \mathrm{~mL}$ volumetric flask and brought to the final volume using $\mathrm{CH}_{2} \mathrm{Cl}_{2}$ for instrumental analysis.
GC/MS analysis: Evaluation of the solid-phase extraction method and concentration measurements in aqueous samples was performed by GC/MS. The GC system was equipped with a cold on-column injector allowing liquid injections as well as solid-phase microextraction followed by thermal desorption. Helium was used as the carrier gas and the column head pressure was maintained at $10 \mathrm{psi}$ to give an approximate flow rate of $1 \mathrm{~mL} \mathrm{~min}^{-1}$. The column temperature was initially held at $70^{\circ} \mathrm{C}$ for $4 \mathrm{~min}$, ramped to $300{ }^{\circ} \mathrm{C}$ at a rate of $10{ }^{\circ} \mathrm{C} / \mathrm{min}$ and then held at $300{ }^{\circ} \mathrm{C}$ for $10 \mathrm{~min}$. The mass spectrometer detector was used in the electron ionization mode and all spectra were acquired using a mass range of $50-400 \mathrm{~m} / \mathrm{z}$ and automatic gain control.

GC/C-IRMS analysis: Stable carbon isotope measurements of individual polycyclic aromatic hydrocarbons were carried out using TraceQ gas chromatograph equipped with a split/splitless injector interfaced with a combustion furnace to a MAT253 isotopic mass spectrometer (Finnigan MAT, Bremen, Germany). The combustion interface contained $\mathrm{Cu}$, $\mathrm{Ni}$ and $\mathrm{Pt}$ wires doped with oxygen $(\mathrm{CuO}$ and $\mathrm{NiO})$ and maintained at $960^{\circ} \mathrm{C}$. Co-generated water vapor was removed using a Nafion elimination trap and interfering nitrogen oxides were reduced to $\mathrm{N}_{2}$ gas through a $600{ }^{\circ} \mathrm{C}$ reduction column. The purified $\mathrm{CO}_{2}$ was swept into the isotope ratio mass spectrometer, where the carbon isotope composition was measured using isodat 3 software. The combustion interface was operated as described by Merritt et al. ${ }^{25}$. The initial GC conditions were similar to those used for GC-MS. The GC method was modified by changing the carrier gas flow, column head pressure, purge time, oven temperature program, etc., to achieve optimal separation of polycyclic aromatic hydrocarbons from other interfering compounds on the GC/C-IRMS system. Injections $(1 \mu \mathrm{L})$ were made in the splitless mode $(50 \mathrm{~s})$ and the injector temperature was maintained at $275^{\circ} \mathrm{C}$. The column temperature started at $70{ }^{\circ} \mathrm{C}$ for $1.5 \mathrm{~min}$ and increased to $140{ }^{\circ} \mathrm{C}$ at a rate of $12{ }^{\circ} \mathrm{C} / \mathrm{min}$. The rate was then increased to $6{ }^{\circ} \mathrm{C} / \mathrm{min}$ until $170{ }^{\circ} \mathrm{C}$ was reached and held for $11 \mathrm{~min}$. The rate was further increased to $10{ }^{\circ} \mathrm{C} / \mathrm{min}$ until $310^{\circ} \mathrm{C}$ was reached and held for $3 \mathrm{~min}$. The flow rate of the carrier gas $(\mathrm{He})$ was $1 \mathrm{~mL} / \mathrm{min}$. Prior to injection, the carbon dioxide reference gas with a known isotopic ratio and a standard mixture containing 10 $n$-alkanes with known isotopic composition (provided by University of Indiana, Bloomington, USA), was injected three times per day during the measuring period to monitor the reliability of background subtraction. $\delta^{13} \mathrm{C}$ values were calculated as

$$
\delta^{13} \mathrm{C}=\left[\frac{\mathrm{R}_{\mathrm{S}}}{\mathrm{R}_{\mathrm{std}}}-1\right] \times 1000(\%)
$$

where $\delta^{13} \mathrm{C}$ is the stable carbon isotope ratio, $\mathrm{R}$ is the ${ }^{13} \mathrm{C} /{ }^{12} \mathrm{C}$ for stable carbon, $\mathrm{s}$ is the sample and std is the standard. Samples were referenced against $\mathrm{CO}_{2}$ calibrated to Vienna PeeDee Belmenite. All peak voltages were well within instrument linearity (1-6 V), or were discarded in subsequent analysis. Isotopic analyses were performed in the open laboratory of groundwater and engineering, IHEG, Zheng Ding, He Bei, China.

\section{RESULTS AND DISCUSSION}

The solid-phase extraction procedure can purify the extraction of water samples prior to analysis by GC and reduce 
interference peaks in the analysis of polycyclic aromatic hydrocarbon chromatograms. The integrity of the samples during extraction, purification and instrumental analysis was verified with a quantitative mass balance of all analytes of interest by the GC/MS system. Table-1 shows the results of the GC/MS method validation for the investigated standard solutions with individual polycyclic aromatic hydrocarbons. The linearity of the SPE-GC/MS method was tested $\left(r^{2}=0.995\right)$ within the concentration range of $0.05-50 \mu \mathrm{g} \mathrm{L}^{-1}$. Analyte recoveries reflecting the accuracy of the solid-phase extraction method and the recoveries of the 16 polycyclic aromatic hydrocarbons for standard solutions after all purification steps were 85.8 to $112 \%$ (average $=95 \%$ ). With relative standard deviations (RSDs) of $=10 \%$ (average $4.5 \%$ ) in all recovery tests, the solid-phase extraction method proved to be the highly accurate and precise. In most cases, low recoveries were obtained (particularly for heavy polycyclic aromatic hydrocarbons with more rings) because increased ring number of polycyclic aromatic hydrocarbons also increased the interaction between polycyclic aromatic hydrocarbons and C18. Therefore, polycyclic aromatic hydrocarbons adsorbed on C18 were more difficultly eluted and resulted in lower recovery. On the other hand, some factors can lead to high recoveries (even up to $112 \%$ ), such as matrix effect, operation errors, method errors, etc., although the recoveries are still acceptable.

Precision and accuracy of $\boldsymbol{\delta}^{13} \mathbf{C}$ measurement: To evaluate the performance of the SPE-GC/C-IRMS system, $\delta^{13} \mathrm{C}$ standard values for the 16 polycyclic aromatic hydrocarbons were measured using processed and unprocessed standard materials and the results are shown in Table-2. The standard deviation (SD) of the IRMS method was between 0.44 and 3.03 for 6 measurements not including dibenzo $[b, c]$ fluoranthene and benzo[ $k]$ fluoranthene. The rest of the SD ranged from 0.44 to 1.02. The deviations of $\delta^{13} \mathrm{C}$ signatures from the reference values were $<0.7 \%$. The consistency of the methods suggested that solid-phase extraction coupled with GC/C-IRMS can be used to measure the $\delta^{13} \mathrm{C}$ value of individual polycyclic aromatic hydrocarbons with high accuracy.

TABLE-1

ANALYTICAL PERFORMANCES OF THE DEVELOPED SPE-GC/MS METHOD

\begin{tabular}{|c|c|c|c|c|c|}
\hline S. No. & Compound & Retention time (min) & Recovery & Regression coefficient & $\operatorname{RSD}(\%)$ \\
\hline 1 & Naphthalene & 8.4 & 92.3 & 0.994 & 4.60 \\
\hline 2 & Acenaphthylene & 12.6 & 87.7 & 0.997 & 1.70 \\
\hline 3 & Acenaphthene & 13.1 & 90.8 & 0.996 & 0.89 \\
\hline 4 & Fluorine & 13.9 & 102.5 & 0.995 & 6.30 \\
\hline 5 & Phenanthrene & 16.0 & 97.2 & 0.997 & 6.59 \\
\hline 6 & Anthracene & 16.2 & 89.8 & 0.996 & 4.98 \\
\hline 7 & Fluoranthene & 24.88 & 100.4 & 0.998 & 3.40 \\
\hline 9 & Benz[a]anthracene & 29.00 & 91.2 & 0.999 & 4.21 \\
\hline 10 & Chrysene & 29.26 & 85.8 & 0.998 & 1.68 \\
\hline 11 & Benzo[b]fluoranthene & 33.25 & 82.0 & 0.997 & 1.86 \\
\hline 12 & Benzo $[k]$ fluoranthene & 33.34 & 80.7 & 0.996 & 2.53 \\
\hline 13 & Benzo[a]pyrene & 34.40 & 105.9 & 0.997 & 2.12 \\
\hline 14 & Ideno[ $[1,2,3-c d]$ pyrene & 38.34 & 87.2 & 0.995 & 10.9 \\
\hline 15 & Dbenz $[a, h]$ anthracene & 38.56 & 87.8 & 0.997 & 12.9 \\
\hline
\end{tabular}

TABLE-2

COMPARISION OF $\delta^{13} \mathrm{C}_{\mathrm{V}-\mathrm{PDB}}(\%)$ VALUES BY PURE PHASE STANDARD MATERIAL AND PROCESSED STANDARD MATERIAL

\begin{tabular}{|c|c|c|c|}
\hline \multirow{2}{*}{ Compound name } & \multicolumn{3}{|c|}{$\delta^{13} \mathrm{C}$ values $(\%)^{\mathrm{a}}$} \\
\hline & After solid-phase extraction & Pure liquid phase & $\Delta \delta^{13} \mathrm{C}$ values \\
\hline Naphthalene & $-26.23 \pm 0.58(n=6)$ & $-26.03 \pm 0.22(n=8)$ & -0.17 \\
\hline Acenaphthylene & $-23.82 \pm 0.44(n=6)$ & $-23.50 \pm 0.30(n=8)$ & -0.32 \\
\hline Acenaphthene & $-24.11 \pm 0.63(n=6)$ & $-24.41 \pm 0.33(n=8)$ & 0.3 \\
\hline Fluorine & $-28.24 \pm 0.70(n=6)$ & $-28.44 \pm 0.50(n=8)$ & 0.2 \\
\hline Phenanthrene & $-27.45 \pm 0.88(n=6)$ & $-27.20 \pm 0.68(n=8)$ & -0.25 \\
\hline Anthracene & $-26.56 \pm 0.54(n=6)$ & $-26.84 \pm 0.24(n=8)$ & 0.28 \\
\hline Fluoranthene & $-28.16 \pm 0.88(n=6)$ & $-28.56 \pm 0.36(n=8)$ & 0.40 \\
\hline Pyrene & $-25.98 \pm 0.94(n=6)$ & $-25.54 \pm .28(\mathrm{n}=7)$ & -0.44 \\
\hline Benz[a]anthracene & $-27.01 \pm 0.96(n=6)$ & $-27.40 \pm 0.46(n=8)$ & 0.39 \\
\hline Chrysene & $-25.22 \pm 1.02(n=6)$ & $-25.40 \pm 0.22(n=8)$ & 0.18 \\
\hline Benzo[b]fluoranthene & $-27.88 \pm 3.03(n=6)$ & $-27.01 \pm 0.53(n=8)$ & -0.87 \\
\hline Benzo $[k]$ fluoranthene & $-25.23 \pm 2.01(n=6)$ & $-26.60 \pm 0.60(n=8)$ & 1.37 \\
\hline Benzo[a]pyrene & $-26.43 \pm 0.88(n=6)$ & $-26.08 \pm 0.35(n=8)$ & -0.35 \\
\hline Dibenz $[a, h]$ anthracene & $-23.66 \pm 0.89(n=6)$ & $-24.26 \pm 0.33(n=8)$ & 0.60 \\
\hline Benzo[ghi]perylene & $-27.82 \pm 1.00(n=6)$ & $-27.47 \pm 0.45(n=8)$ & -0.35 \\
\hline
\end{tabular}


Effect of solid-phase extraction on the $\delta^{13} \mathrm{C}$ values of individual polycyclic aromatic hydrocarbons: To determine whether isotope effects occur during extraction, the $\delta^{13} \mathrm{C}$ values of a mixture containing polycyclic aromatic hydrocarbon standard solutions after solid-phase extraction procedure were compared with pure liquid-phase materials.

The pure liquid phase of the 16 polycyclic aromatic hydrocarbons standards dissolved in $n$-hexane was measured by direct injection into the GC-C/IRMS system for the correction of $\delta^{13} \mathrm{C}$ values of the sample analytes. From this solution, $1 \mathrm{~mL}$ was injected to run in split mode at a split flow of $1 \mathrm{~mL}$ $\mathrm{min}^{-1}$. To protect the coating of the pyrolysis reactor, the incoming $n$-hexane was removed by activating the backflush mode. The measurement was performed according to GC/CIRMS analysis method in triplicates. The $\delta^{13} \mathrm{C}$ values of individual polycyclic aromatic hydrocarbons in the environmental samples were obtained from two repeated analyses and the results were expressed using the mean values of the two repeated measurements. The analytical method was evaluated to ensure that the compositional and stable isotopic integrity was preserved throughout the extraction scheme, by processing standards with known stable isotopic compositions. The results of stable carbon isotopic measurements of the standard materials after purification were compared with those of unprocessed standard materials (Fig. 1). A diagonal line represented a 1:1 ratio between the isotope values of processed and unprocessed standard materials. Almost all obtained $\delta^{13} \mathrm{C}$ values fell on the diagonal line, which well agreed with the isotope signatures measured during extraction. O'Malley et al. ${ }^{7}$ demonstrated that evaporation had no significant effect on the measured stable carbon isotope ratios of polycyclic aromatic hydrocarbons. Using the solid-phase extraction method, deviations in $\delta^{13} \mathrm{C}$ signatures from the reference values were lower, except for dibenzo[b,c]fluoranthene and benzo[k]fluoranthene, wherein the $\Delta \delta^{13} \mathrm{C}_{\text {SPE-standard }}$ was between 0.87 and $1.37 \%$ (Table-2). We did not observe any systematic trend for a method dependent on $\mathrm{C}$ isotope fractionation using SPE-GC/IRMS. This observation suggested the occurrence of isotope fractionation during SPE-GC/IRMS analysis, which was either associated with the conversion of analytes during oxidation or reduction in the combustion interface or with analyte extraction by SPE. Several reasons can explain the slight enrichment. First, isotopic fractionation during the concentration steps may have caused a preferential loss of isotopically light polycyclic aromatic hydrocarbons during evaporation. Physicochemical processes such as evaporation involves an isotope exchange effect that results in preferential enrichment of lighter molecular species in the vapor phase and enrichment of heavier molecules in the residue $^{22}$. Second, these compounds exhibit different chemical properties with regard to their ability to undergo intermolecular interactions (i.e, van der waals vs. electron donor or electron acceptor interactions) with water and the $\mathrm{C} 18$ disk. The three sources, which are either associated with the conversion of the analytes during oxidation or reduction in the combustion interface, or with analyte extraction by solid-phase extraction, as well as other processes, may also induce minor changes in carbon isotope compositions for some compounds. Furthermore, dibenzo $[b, c]$ fluoranthene and benzo $[k]$ fluoranthene are

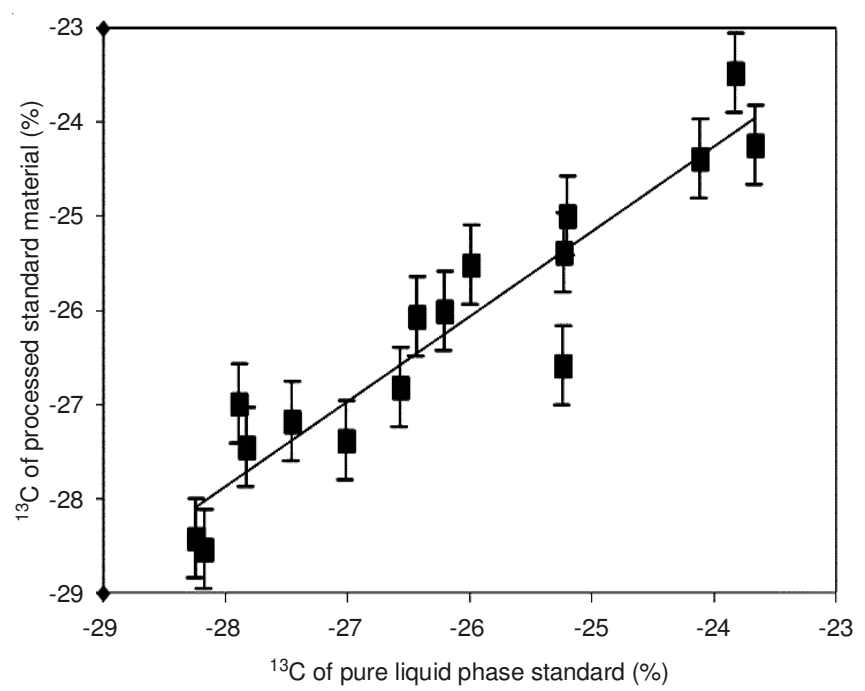

Fig. 1. Relationship of $\delta^{13} \mathrm{C}(\%)$ of polycyclic aromatic hydrocarbons between pure liqiuid standard material and processed standard material; Note: Refer to (Table-1) for abbreviation, compounds listed from left to right on the $\mathrm{x}$-axis are fluorene, fluoranthene, dibenzo $[b, c]$ fluoranthene, benzo[ $g h i]$ perylene, phenanthrene, anthracene, benzo[a]pyrene, naphthalene, pyrene, benzo[k]fluoranthene, chrysene, indeno[1,2,3cd]pyrene, acenaphthene, acenaphthylene, dibenz $[a, h]$ anthracene

isomeric chemical compounds exhibiting similar interaction properties that make complete separation using chromatography difficult. We speculate that the reduction of carbon species to $\mathrm{CO}_{2}$ in the reduction furnace may have been incomplete and that isotope fractionation occurred during oxidation of the analyte or during the reduction of partially oxidized C species.

In this study, even though slight enrichments occurred for some compounds, the differences were minimal and almost all isotope ratios of individual polycyclic aromatic hydrocarbons for the purified standard material fell within the two standard deviations $(2 \sigma)$ of the mean isotope ratios of the unprocessed standard materials.

\section{Conclusion}

A method of solid-phase extraction coupled with GC/CIRMS for the measurement of $\delta^{13} \mathrm{C}$ of polycyclic aromatic hydrocarbons in aqueous samples was developed. The purification and isotope analysis methods can effectively and accurately measure the stable carbon isotope ratios of polycyclic aromatic hydrocarbons in aqueous-phase samples. For the first time, an solid-phase extraction-based method was evaluated in detail for compound-specific stable carbon isotope analysis. This technique greatly enhanced the efficiency of isotope analysis by eliminating certain procedures and reducing the chance of isotopic fractionation. The development of this technique for $\mathrm{C}$ stable isotopes can be used extensively to determine contaminant sources and understanding the mechanisms underlying polycyclic aromatic hydrocarbon formation in water contamination sites.

\section{ACKNOWLEDGEMENTS}

This work was financially supported by the Fundamental Research Funds for the IHEG, No. SK201101. 


\section{REFERENCES}

1. R.M. Marce and F. Borrull, J. Chromatogr. A, 885, 273 (2000).

2. R. Niessner, D. Klockow, F. Bruynseels and R. Van Grieken, Anal. Chem., 22, 281 (1985).

3. J.M. Bayona, J. Albaiges, A.M. Solanas, R. Pares, P. Garrigues and M. Ewald, Anal. Chem., 23, 289 (1986).

4. G.S. Douglas, A.E. Bence, R.C. Prince, S. McMillen and E.L. Butler, Environ. Sci. Technol., 30, 2332 (1996)

5. F.D. Hostettler and K.A. Kvenvolden, Org. Geochem., 21, 927 (1994).

6. Z. Wang and M. Fingas, J. Chromatogr. A, 712, 321 (1995).

7. O'Malley, Ph.D .Thesis, Compound-Specific Carbon Isotope Geochemistry of Polycyclic Aromatic Hydrocarbons in Eastern Newfoundland Estuaries. Memorial University of Newfoundland (1994).

8. D.C. Ballentine, S.A. Macko, V.C. Turekian, W.P. Gilhooly and B. Martincigh, Org. Geochem., 25, 97 (1996).

9. B.T. Hammer, C.A. Kelley, R.B. Coffin, L.A. Cifuentes and J.G. Mueller, Chem. Geol., 152, 43 (1998).

10. C. McRae, C.G. Sun, C.E. Snape, A. Fallick and D. Taylor, Org. Geochem., 30, 881 (1999).

11. C. McRae, C.E. Snape, C.G. Sun, D. Fabbri, D. Tartari, C. Trombini and A. Fallick, Environ. Sci. Technol., 34, 4684 (2000).

12. L. Mazeas and H. Budzinski, J. Chromatogr. A., 923, 165 (2001).

13. D. Hunkeler, R. Aravena, K. Berry-Spark and E. Cox, Environ. Sci. Technol., 39, 5975 (2005).
14. D. Hunkeler, N. Chollet, X. Pittet, R. Aravena, J.A. Cherry and B.L.J. Parker, J. Contam. Hydrol., 74, 265 (2004).

15. L. Zwank, M. Berg, T.C. Schmidt and S.B. Haderlein, Anal. Chem., 75, 5575 (2003).

16. K. Yamada, N. Yoshida, G. Calderone and C. Guillou, Commun. Mass Spectrom., 21, 1431 (2007).

17. M. Berg, J. Bolotin and T.B. Hofstetter, Anal. Chem., 79, 2386 (2007).

18. D.L. Song, M.E. Conrad, K.S. Sorenson and L. Alvarez-Cohen, Sci. Technol., 36, 2262 (2002)

19. M.A. Jochmann, M. Blessing, S.B. Haderlein and T.C. Schmidt, Rapid Commun. Mass Spectrom., 20, 3639 (2006).

20. J. Pawliszyn, Solid Phase Microextraction-Theory and Practice, WileyVCH, New York (1997).

21. A.L. Norman, J.F. Hopper, P. Blanchard, D. Ernst, K. Brice, N. Alexandrou, G. Klouda, Atmos. Environ., 33, 2807 (1999).

22. T. Okuda, H. Kumata, H. Naraoka, R. Ishiwatari and H. Takada, Org. Geochem., 33, 843 (2002).

23. T. Okuda, H. Kumata, M.P. Zakaria, H. Naraoka, R. Ishiwatari and H. Takada, Atmos. Environ., 36, 611 (2002).

24. M.M.G. Chartrand, P.L. Morrill, G. Lacrampe-Couloume and B. Sherwood Lollar, Environ. Sci. Technol., 39, 4848 (2005).

25. D.A. Merritt, F.H. Freeman, M.P. Ricci, S.A. Studley and J.M. Hayes, Anal. Chem., 67, 2461 (1995). 